\title{
Structural Comparison between the Right and Left Atrial Appendages Using Multidetector Computed Tomography
}

\author{
Koichi Shinoda, ${ }^{1,2}$ Shogo Hayashi, ${ }^{3}$ Daisuke Fukuoka, ${ }^{4}$ Ryo Torii, ${ }^{2,5}$ \\ Tsuneo Watanabe, ${ }^{1}$ and Takashi Nakano ${ }^{2}$ \\ ${ }^{1}$ Department of Clinical Laboratory, Gifu University Hospital, 1-1 Yanagido, Gifu-shi, Gifu 501-1194, Japan \\ ${ }^{2}$ Department of Anatomy, Aichi Medical University, 1-1 Yazakokarimata, Nagakute-shi, Aichi 480-1195, Japan \\ ${ }^{3}$ Department of Anatomy, Tokyo Medical University, 6-1-1 Shinjuku, Shinjuku-ku, Tokyo 160-8402, Japan \\ ${ }^{4}$ Research and Development Center for Human Medical Engineering, Gifu University, 1-1 Yanagido, Gifu-shi, Gifu 501-1194, Japan \\ ${ }^{5}$ Department of Physical Therapy, Chubu Rehabilitation College, 2-2 Wakamiya-cho, Nakamura-ku, \\ Nagoya-shi, Aichi 453-0023, Japan \\ Correspondence should be addressed to Shogo Hayashi; sho5-884@umin.ac.jp
}

Received 26 June 2016; Revised 5 October 2016; Accepted 16 October 2016

Academic Editor: Christof Kolb

Copyright (C) 2016 Koichi Shinoda et al. This is an open access article distributed under the Creative Commons Attribution License, which permits unrestricted use, distribution, and reproduction in any medium, provided the original work is properly cited.

The three-dimensional (3D) structures of the right atrial appendage (RAA) and left atrial appendage (LAA) were compared to clarify why thrombus formation less frequently occurs in RAA than in LAA. Morphological differences between RAA and LAA of 34 formalin-preserved cadaver hearts were investigated. Molds of RAA and LAA specimens were made and the neck areas, volumes of the atrial appendages (AA), and amount of pectinate muscles (PMs) were analyzed using multidetector computed tomography. In RAA, most PMs were connected to one another and formed a "dendritic" appearance and the inner surface area was smaller than in LAA. RAA had smaller volumes and larger neck areas than LAA. The ratios of the neck area/volume were larger and the amounts of PMs were smaller in RAA than in LAA. The volumes, neck areas, and amount of PMs of RAA were significantly correlated with those of LAA. According to the 3D structure, RAA appears to be suited for a more favorable blood flow, which may explain why the thrombus formation is less common in RAA than in LAA. Examining not only LAA but also RAA by transesophageal echocardiography may be useful in high-risk patients of thrombus formation in LAA because the volume, neck area, and amount of PMs of LAA reflect the shape of RAA.

\section{Introduction}

The atrial appendage (AA) is embryologically derived from the primary atrium and protrudes as an ear-shaped part of the atrium [1]. The inner surfaces of AA are characterized by muscular ridges, that is, the pectinate muscles (PMs). Furthermore, thrombi are frequently formed within AA, which is also the site of atrial natriuretic peptide secretion in mammals $[2,3]$. Moreover, AA is considered to modulate the association between atrial pressure and volume. Thus, AA is considered important in the states of atrial volume overload $[4,5]$.

Clinically, thrombi are more frequently formed in the left atrial appendage (LAA) than in the right atrial appendage (RAA) among patients with atrial fibrillation (AF) [6-13].
Thus, the frequency of thrombus formation is believed to be related to the differences between the luminal surface area and the size of the neck between RAA and LAA [14].

Although a few studies have attempted to compare the structures and functions of RAA and LAA by transesophageal echocardiography (TEE) $[4,6,7,14]$, detailed anatomical differences remain unclear, possibly because the resolution of cardiac ultrasonography is too low to reveal the internal fine structures of AA, for example, PMs. Furthermore, the volume and shape of LAA greatly vary according to the imaging plane [15]. It has been reported that greater bending and spiraling of LAA are associated with a greater variance in images that are obtained from different two-dimensional (2D) planes [16]. Therefore, elucidating the morphological details and accurate 
observations of AA is difficult by cardiac ultrasound inspection.

Although some studies have described the structure of LAA using molds of gross anatomical structures, none have mentioned RAA. In addition, the usefulness of multidetector computed tomography (MDCT) has been recently established for depicting vascular structures and, thus, is now considered a feasible imaging modality to select a surgical method and for preoperative planning [17]. However, to the best of our knowledge, no previous study has examined the differences in three-dimensional (3D) structures between RAA and LAA. Thus, this study aimed to hybridize these methods (i.e., molds and MDCT images) to analyze morphological and quantitative differences between RAA and LAA.

\section{Materials and Methods}

The hearts of 34 formalin-preserved cadavers (11 males and 23 females; mean age at death, $84 \pm 8.4$ years), which were donated to the Aichi Medical University, were dissected. The cadavers with a cause of death of embolism or valvular heart disease were excluded.

AA was dissected in a manner that preserved some of the atrial wall to identify morphological differences between RAA and LAA, particularly gross anatomical differences in PMs. Then, silicon molds of AA from each heart were produced, which included a portion of the neck between RAA and LAA (silicone acrylic hybrid resin; Nissin Chemical Industry Co., Ltd., Echizen, Japan; mixing ratio of resin to hardener, 100:5 w/w; curing time, 7 days). The neck portion of RAA and LAA was defined as the entrance part of the AA for measurement using TEE $[7,18]$. The RAA was measured in conjunction with the two parts divided by the tenia sagittalis. Each mold was inspected using MDCT of a Philips Brilliance 64-slice CT system (Royal Philips, Amsterdam, Netherlands). CT scanning protocols: tube voltage, $120 \mathrm{kVp}$; tube current, $50 \mathrm{~mA}$; detector configuration, 64-row detectors with $0.625 \mathrm{~mm}$ section thickness; beam collimation, $40 \mathrm{~mm}$; rotation time, $0.5 \mathrm{~s}$; pitch, 0.515 ; display field of view, $16 \mathrm{~cm}$; imaging filter, C-types (Figure 1).

2.1. Mold Measurements. For each mold, the volumes, neck areas, and surface areas of AAs were measured. After binarycoded processing was performed for all volume data in the MDCT image, we performed 3D labeling processing and decided a domain of the whole AA. Computer analysis of overall volume from the number of voxel and neck and surface areas from the number of pixels was performed. From these measurements, the neck area/volume ratio and surface area/volume ratio for each AA were calculated. Furthermore, we excised PMs from each AA by 3D morphological operations (Figure 2) [19] and quantified differences between RAA and LAA. The amount of PMs was determined from the number of voxels using computer analysis.

2.2. Statistical Analyses. All measurements are presented as means \pm standard deviations. Differences between RAA and
LAA were compared using two-tailed paired $t$-tests and Pearson's product moment correlation coefficient. A probability $(p)$ value of $<0.05$ was considered statistically significant.

\section{Results}

3.1. Gross Anatomy of RAA and LAA. The inner surfaces of both RAA and LAA are characterized by PMs. There are significant differences between RAA and LAA; as in RAA, most PMs are connected to one another and form a "dendritic" appearance. In contrast, connections between neighboring PMs are less pronounced in LAA (Figure 3).

3.2. Neck Areas of RAA and LAA. The mean neck area of LAA was significantly smaller than that of RAA (Table 1). There was a distinct correlation between the neck areas of RAA and LAA (Figure 4(a)).

3.3. Volumes of RAA and LAA. The mean volume of LAA was significantly greater than that of RAA (Table 1). There existed a distinct correlation between the volumes of RAA and LAA (Figure 4(b)).

3.4. Correlation between Neck Areas and Volumes. There was a significant correlation between the volume of LAA and the neck area of LAA (Figure 5(a)). However, such a significant correlation was not present in RAA (Figure 5(b)).

3.5. Neck Area/Volume Ratios of RAA and LAA. The mean neck area/volume ratio of LAA was significantly smaller than that of RAA (Table 1). There was no significant correlation between the neck area/volume ratios of RAA and LAA (Figure 4(c)).

3.6. Surface Area/Volume Ratios of RAA and LAA. The mean surface area/volume ratio of LAA was significantly greater than that of RAA (Table 1). There was no significant correlation between the surface area/volume ratios of the RAA and LAA (Figure 4(d)).

3.7. Amount of PMs in RAA and LAA. The mean amount of PMs of LAA was significantly greater than that of RAA (Table 1). There existed a distinct correlation between the amounts of PMs of RAA and LAA (Figure 4(e)).

\section{Discussion}

There are few reports on the detailed structures of RAA and LAA, particularly anatomical differences. To the best of our knowledge, this is the first study to compare the morphological characteristics of RAA and LAA.

Regarding the gross anatomy of these structures, LAA is a long, tubular, hooked structure that is usually crenelated with a narrow junction within the venous component of the atrium. In contrast, RAA is broad and triangular with a wide junction. Both RAA and LAA are trabeculated with PMs 
TABLE 1: Measurements of RAA and LAA.

\begin{tabular}{|c|c|c|c|}
\hline Measurement part & & RAA & LAA \\
\hline \multirow{3}{*}{ Volume $\left(\mathrm{mm}^{3}\right)$} & Mean \pm SD & $2685 \pm 1610$ & $4375 \pm 2507$ \\
\hline & Range & $557-6618$ & $460-9074$ \\
\hline & $p$ value & \multicolumn{2}{|c|}{$p<0.01$} \\
\hline \multirow{3}{*}{ Neck area $\left(\mathrm{mm}^{2}\right)$} & Mean \pm SD & $425 \pm 313$ & $238 \pm 145$ \\
\hline & Range & 59-1361 & $34-592$ \\
\hline & $p$ value & \multicolumn{2}{|c|}{$p<0.01$} \\
\hline \multirow{3}{*}{ Neck area/volume $\left(\mathrm{mm}^{2} / \mathrm{mm}^{3}\right)$} & Mean \pm SD & $0.016 \pm 0.005$ & $0.013 \pm 0.005$ \\
\hline & Range & $0.009-0.032$ & $0.004-0.025$ \\
\hline & $p$ value & \multicolumn{2}{|c|}{$p=0.02$} \\
\hline \multirow{3}{*}{ Surface area/volume $\left(\mathrm{mm}^{2} / \mathrm{mm}^{3}\right)$} & Mean \pm SD & $0.999 \pm 0.279$ & $1.283 \pm 0.295$ \\
\hline & Range & $0.497-1.651$ & $0.567-1.712$ \\
\hline & $p$ value & \multicolumn{2}{|c|}{$p<0.01$} \\
\hline \multirow{3}{*}{ Amount of pectinate muscles $\left(\mathrm{mm}^{3}\right)$} & Mean \pm SD & $444 \pm 166$ & $514 \pm 217$ \\
\hline & Range & $179-861$ & $128-897$ \\
\hline & $p$ value & \multicolumn{2}{|c|}{$p=0.02$} \\
\hline
\end{tabular}
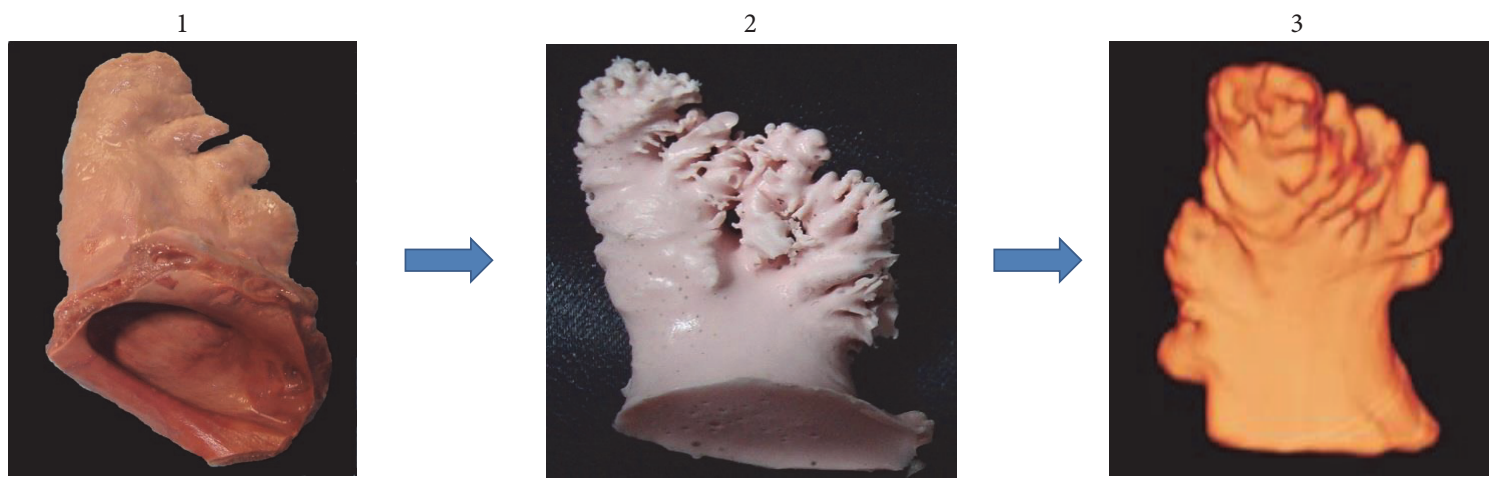

(a)
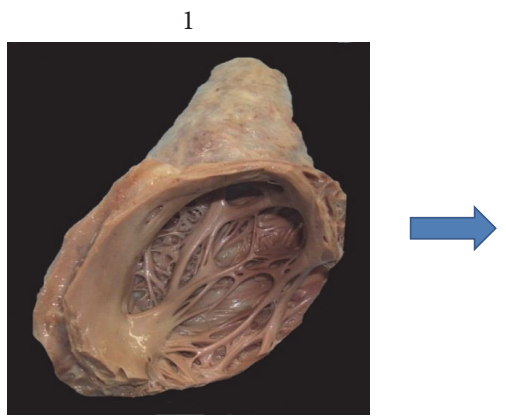
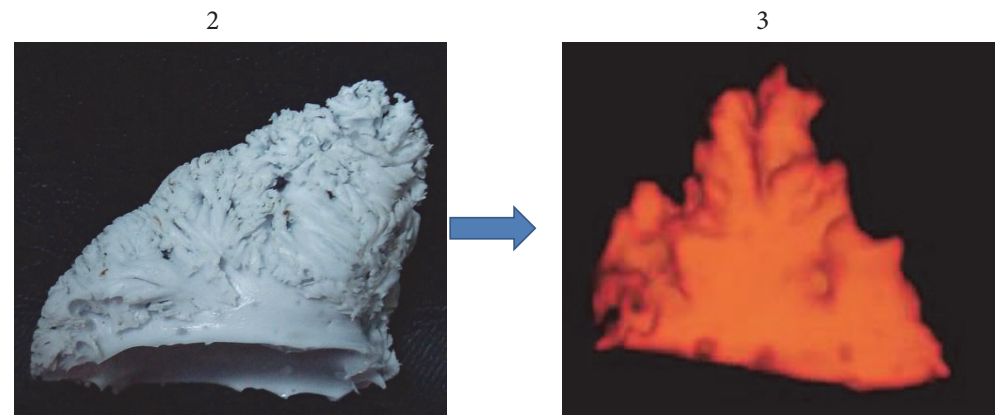

(b)

FIGURE 1: Representative molds of the right atrial appendage (RAA) and the left atrial appendage (LAA). (a)-1 LAA was dissected from the donated body. (a)-2 A silicon mold of LAA. (a)-3 Multidetector computed tomography (MDCT) scanning image of mold of LAA. (b)-1 RAA was dissected from the donated body. (b)-2 A silicon mold of RAA. (b)-3 MDCT scanning image of mold of RAA.

largely running parallel to each other with a comb-like appearance, although these characteristics are less pronounced in LAA [4]. In fact, in this study, some structural differences in PMs were observed between LAA and RAA (Figure 3). In RAA, most of PMs are connected to one another and form a "dendritic" appearance. In contrast, in LAA, connections between neighboring PMs were less pronounced. These morphological characteristics may participate in stagnation of blood flow and may be regarded as a cause of thrombus formation in LAA.

According to Al-Saady et al. [4], the thrombogenic etiology in AA has not been completely elucidated; however, relative stasis, which occurs in AA owing to its shape and inherent PMs, is considered to play a major role. Yamaji et al. [20] 


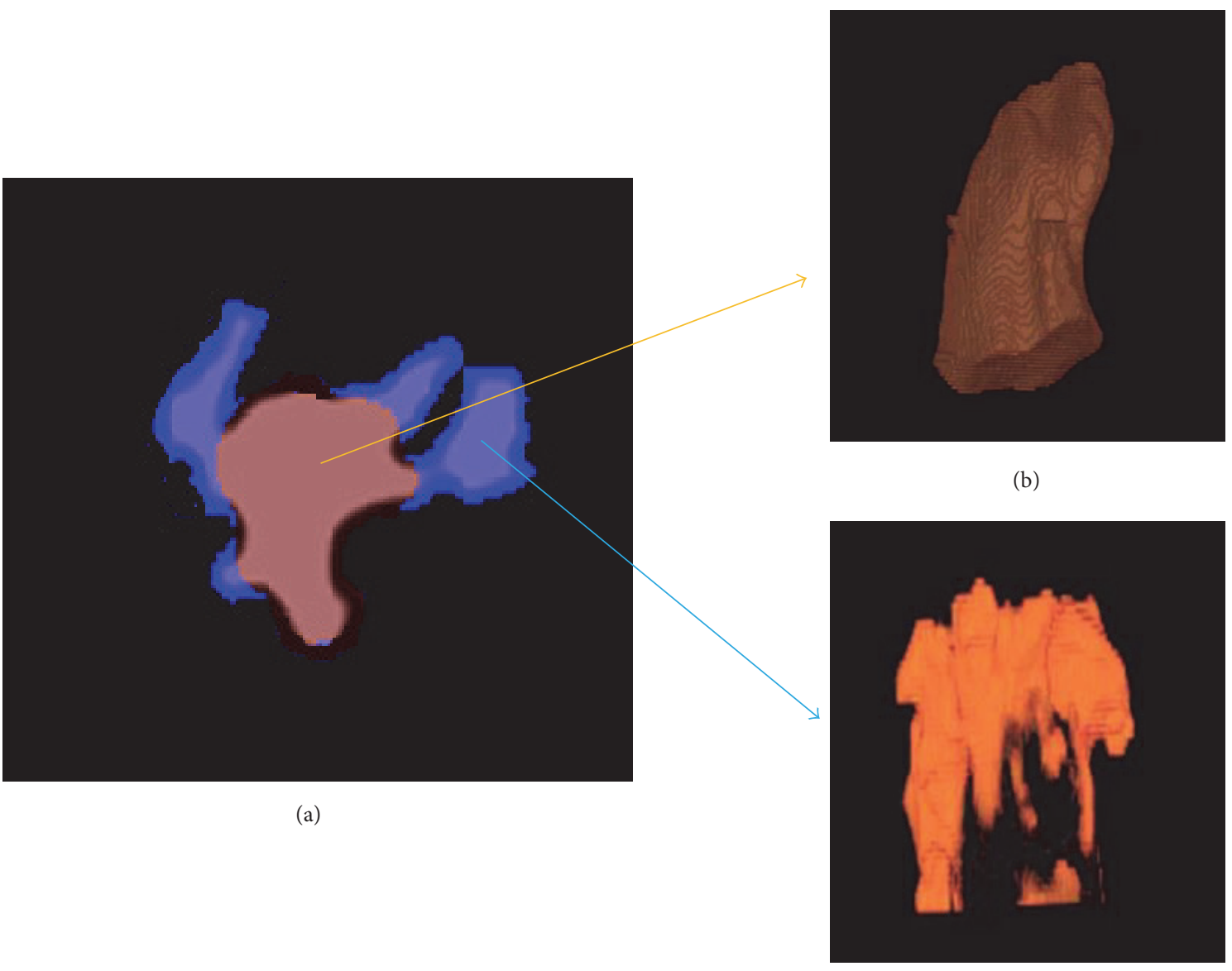

(c)

FIGURE 2: Quantification of the pectinate muscles (PMs) portion of the atrial appendages (AA) by three-dimensional morphological operation. (a) Determination of the PMs portion of the AA by computer analysis (short axis image). (b and c) The PMs were separated from the main body of AA (long axis image). (b) Main body, (c) PMs.

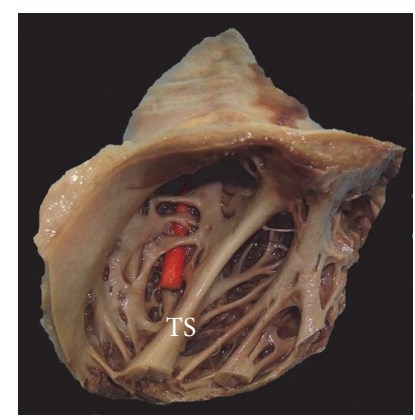

(a)

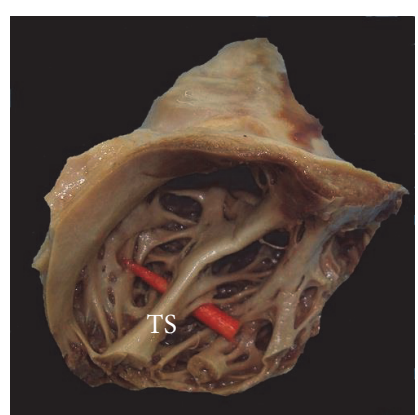

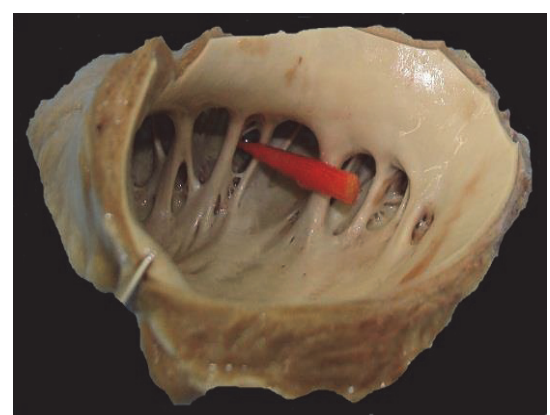

(b)

FIGURE 3: Pectinate muscles (PMs) in right atrial appendage (RAA) and left atrial appendage (LAA). A comparison whether there is intercommunication between PMs. (a)-1,2 PMs in RAA (traffic); (b) PMs in LAA (no traffic). TS: tenia sagittalis.

reported that all thrombi are adhered to PMs. The results of this study revealed a lower incidence of PMs in RAA, indicating that the inner surface area of RAA is smaller than that of LAA. However, there was a distinct positive correlation between the amount of PMs in RAA and that in LAA (Figure 4(e)). Moreover, in RAA, there were fewer PMs
(Table 1), which are the most frequent sites of thrombus formation, than in LAA, which, in terms of both volume and ruggedness, may explain why thrombus formation in RAA less frequently occurs.

RAA had smaller volumes and larger neck areas than LAA, and thus, the mean neck area/volume ratio of RAA 


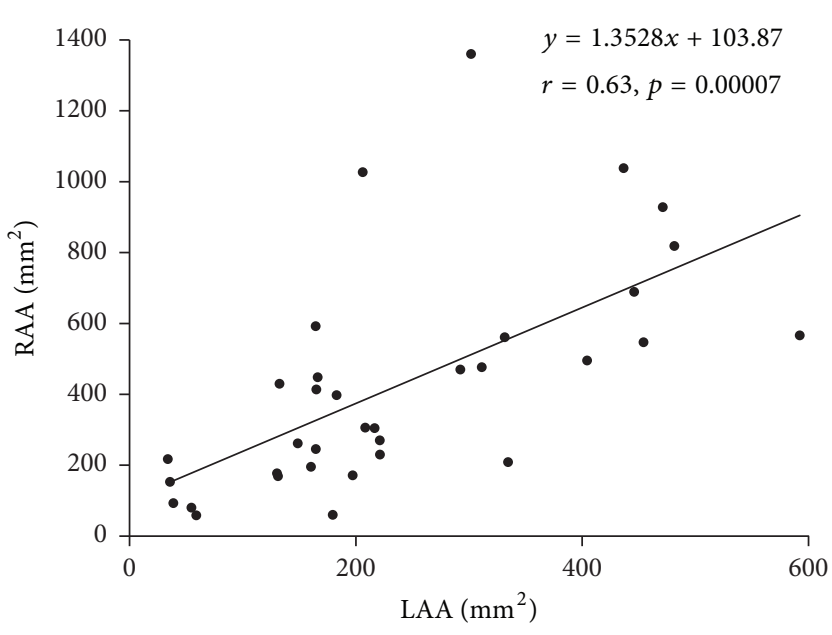

(a)

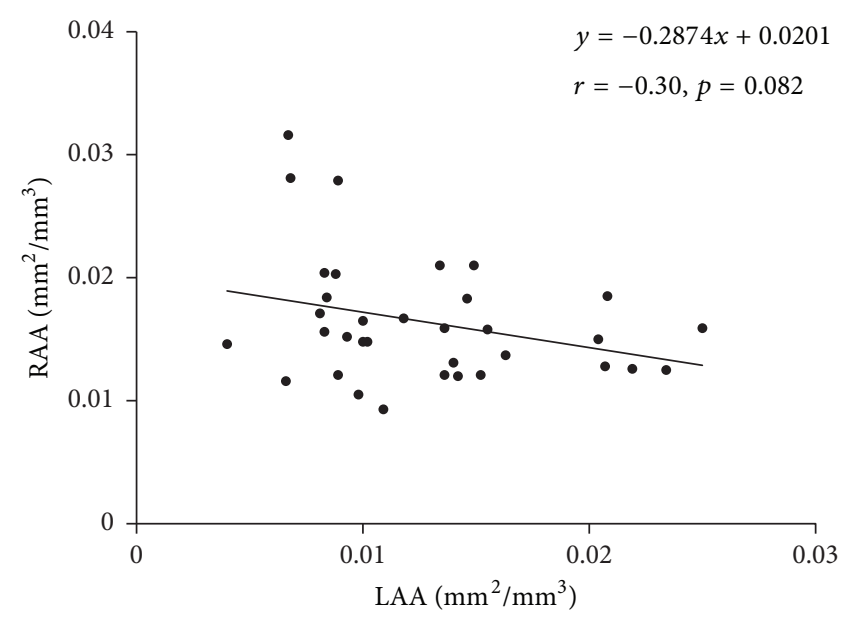

(c)

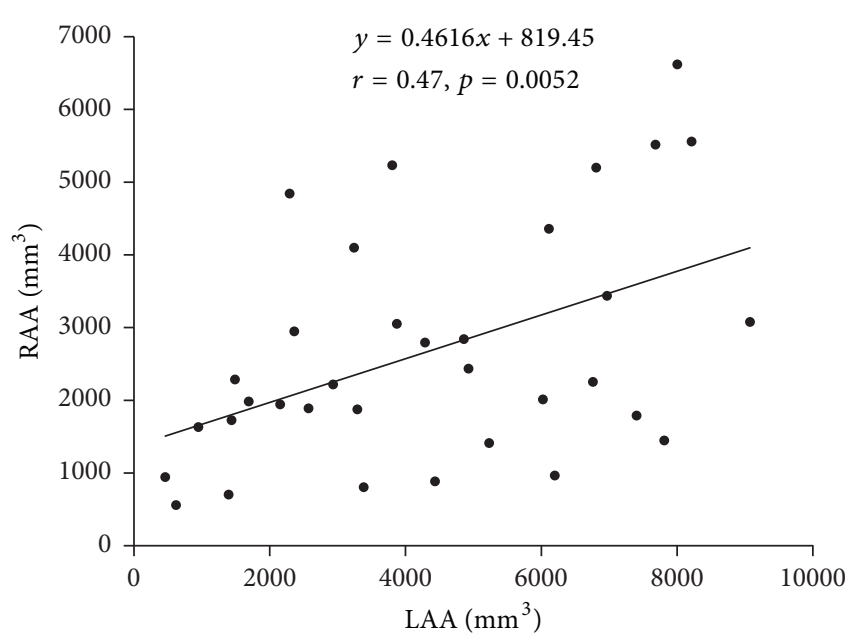

(b)

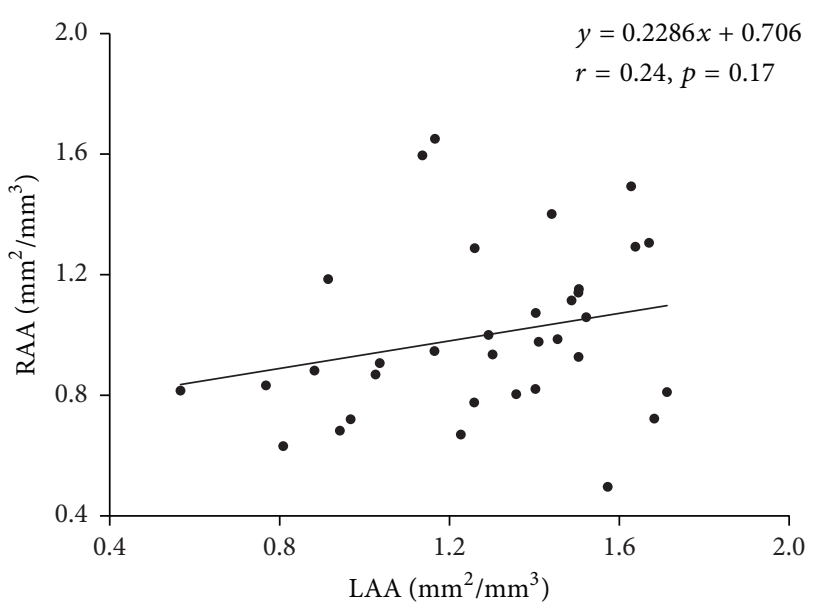

(d)

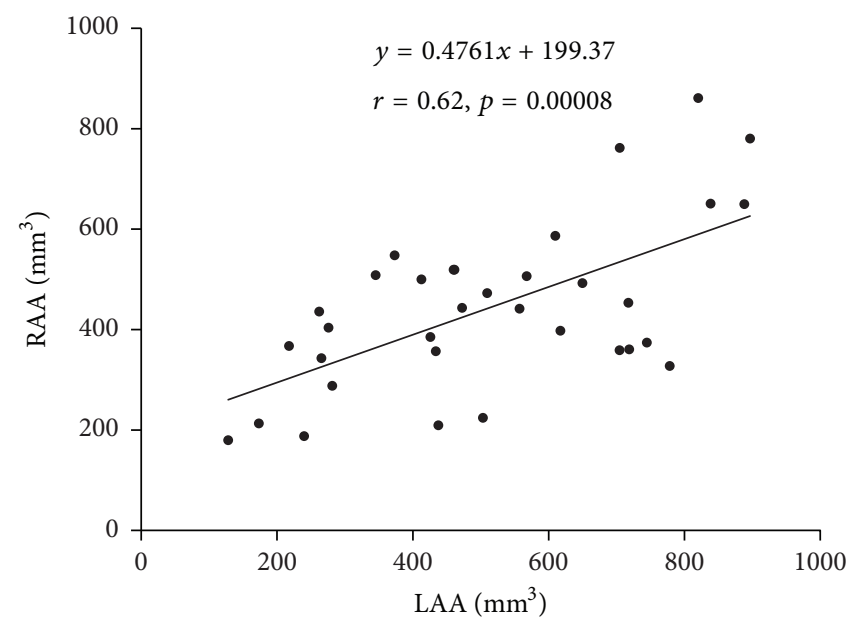

(e)

Figure 4: The correlation between the measured values of right atrial appendage (RAA) and left atrial appendage (LAA). (a) Correlation between the neck areas of RAA and LAA. (b) Correlation between the volumes of RAA and LAA. (c) Correlation between the neck area/volume ratios of RAA and LAA. (d) Correlation between the surface area/volume ratios of RAA and LAA. (e) Correlation between the amount of pectinate muscles (PMs) of RAA and LAA. 


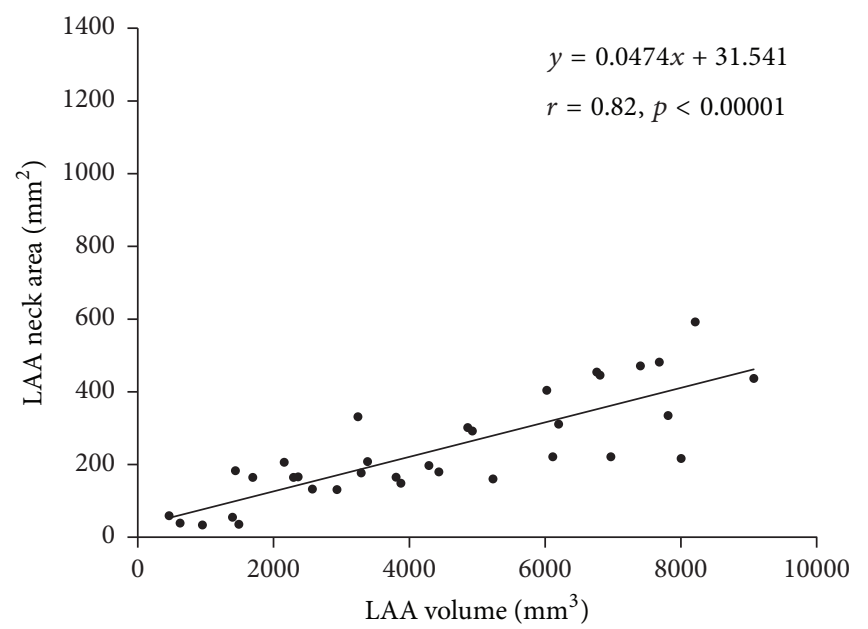

(a)

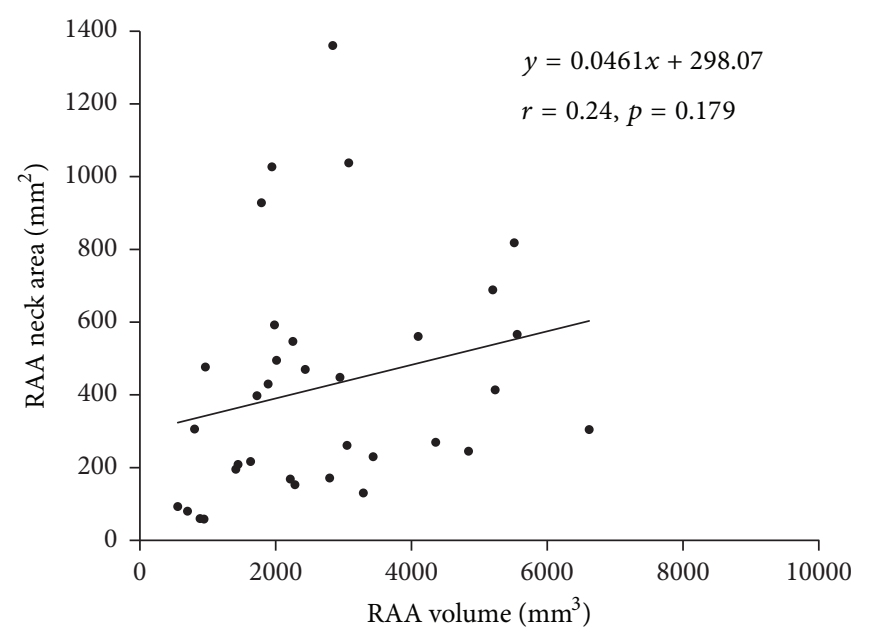

(b)

FIGURE 5: The correlation between the volume and the neck area. (a) Correlation between the volume of left atrial appendage (LAA) and the neck area of LAA. (b) Correlation between the volume of right atrial appendage (RAA) and the neck area of RAA.

was larger (Table 1). If LAA and RAA had similar size and were homothetic, their mean neck area/volume ratio would be similar. Furthermore, if the composition of AA is constant (homothetic) regardless of it, the neck areas have to correlate to the volumes. Actually in LAA, there was a significant correlation between the volume and neck area (Figure 5(a)). However, in RAA there was no significant correlation between them (Figure 5(b)). These results suggest that the composition of LAA may be less variational but the composition of RAA includes applicable variations. The neck areas (Figure 4(a)), volumes (Figure 4(b)), and amounts of PMs (Figure 4(e)) in RAA were significantly correlated with those in LAA. As for the surface area/volume ratio, which can be considered formally smooth, although RAA had significantly larger neck area/volume ratios and smaller surface area/volume ratios than LAA (Table 1), there was no correlation of these parameters between LAA and RAA (Figures 4(c) and 4(d)).

Atrial thrombus formation may occur in various conditions, such as in AF, in valvular heart disease, in response to a mechanical prosthesis, and in restrictive cardiomyopathy $[21,22]$. The present results suggest that thrombus formation within AA may be related to two additional factors, the morphology of AA and the amount of PMs. Atrial thrombi most commonly occur in LAA. According to several clinical studies, RAA thrombi were observed in $0.4 \%-8.3 \%$ of patients with AF, while LAA thrombi were observed in 3.0\%-27.3\% [6-13] (Table 2). Regarding patients with AF, although both the right atrium and the left atrium are fibrillating, the majority of thrombi are located within LAA, while formation in RAA is uncommon. Moreover, thrombus formation generally occurs in the dilated AA [4, 7, 14, 15, 22-24]. Furthermore, Pollick and Taylor [22] reported that LAA thrombus formation was associated with both poor LAA contraction and dilatation, not only in patients with AF but also in those with sinus rhythm. A study by Veinot et al. [25] revealed that the number of lobulations in LAA was not dependent on age or sex; that is, $54 \%$ had at least two lobulations and $80 \%$ had two
TABLE 2: Previous reports of the incidence of thrombus formation in the LAA and RAA in patients with AF.

\begin{tabular}{|c|c|c|c|c|c|c|}
\hline Reference & $N$ & RAA \& LAA & LAA & $\%$ LAA & RAA & $\%$ RAA \\
\hline $\begin{array}{l}\text { Manning et al. } \\
1993\end{array}$ & 94 & 2 & 10 & 11 & 2 & 4.3 \\
\hline $\begin{array}{l}\text { Manning et al. } \\
1995\end{array}$ & 230 & 6 & 34 & 15 & 0 & 2.6 \\
\hline Klein et al. 1997 & 53 & 0 & 6 & 11 & 1 & 1.9 \\
\hline $\begin{array}{l}\text { de Divitiis et al. } \\
1999\end{array}$ & 90 & 5 & 11 & 12 & 1 & 6.7 \\
\hline $\begin{array}{l}\text { Rozenberg et al. } \\
2000\end{array}$ & 230 & NA & 7 & 3 & 1 & 0.4 \\
\hline Bilge et al. 2000 & 53 & 1 & 13 & 25 & 3 & 7.5 \\
\hline Klein et al. 2001 & 549 & NA & 67 & 12 & 9 & 1.6 \\
\hline Sahin et al. 2010 & 84 & NA & 23 & 27 & 7 & 8 \\
\hline
\end{tabular}

or more. Although this study suggested that there exist great variations in the volume and shape of LAA, the measured size of LAA by 2D-TEE may be affected by the cutting plane [25]. The greater extent of bending and spiraling of LAA is associated with a greater variance in images obtained by different $2 \mathrm{D}$ planes. Therefore, this variability should be considered when interpreting images of LAA, particularly when attempting to identify a thrombus [16]. For these reasons, we observed both appendages using $3 \mathrm{D}$ reconstruction with MDCT in this study. On the basis of these findings, it is conceivable that, compared with RAA, LAA has structures that promote stagnation of blood flow.

Thrombus formation may occur less often in RAA than in LAA not only because of contractile dysfunction, as evidenced by a stronger spontaneous contrast echo, and decreased flow rate $[3,18]$, but also because of other causes, such as morphological differences, which we discovered between RAA 
and LAA. Di Biase et al. [26] classified LAA into four categories based on the morphology (cactus, chicken wing, windsock, and cauliflower types) and reported that patients with non-chicken wing LAA morphology are significantly more likely to have an embolic event. Kimura et al. [27] reported that many patients of embolism have the cauliflower type, which suggests that the more complex the internal structure, the more likely the occurrence of thrombus formation. Because the complexity of the AA reported by Di Biase et al. [26] and Kimura et al. [27] is considered to be related to surface area/volume ratio and amount of PMs, they may have an association with thrombus formation. Furthermore, thrombus formation is more likely to occur in LAA as it has a complex morphology due to the high surface area/volume ratio and amount of PMs (Table 1).

Although much clinical attention has focused only on LAA, Ozer et al. [28] and Sahin et al. [13] reported that RAA thrombus formation might be a source of pulmonary embolism. Therefore, although thrombus formation in RAA is a relatively rare event, when compared with LAA, a thrombus in RAA also has the potential to form an embolism and, thus, should also be considered. In addition, we should keep in mind that it is more difficult to visualize RAA anatomy by TEE compared with that of LAA. The incidence of nonvisualization of RAA anatomy during TEE has been reported to be approximately $1.3 \%-16 \%[6,11]$. Moreover, it is next to impossible to visualize the RAA by transthoracic echocardiography, which may also account for the lack of RAA observation. Therefore, we compared the morphological characteristics of RAA and LAA and found significant correlations between the volumes, neck areas, and amounts of PMs. In other words, the structure of LAA formation, which can be comparatively easily investigated by ultrasound, appeared to reflect that of RAA. Although data regarding the formation and occurrence rate of thrombi are limited, observations of not only LAA but also RAA by TEE may be useful in highrisk patients of LAA thrombus formation.

There were several limitations to this study that should be addressed. The first major limitation is the lack of clinical (such as age range and atrial fibrillation) and pathological (such as heart weight, ventricular thickness, chamber dilatation, and previous myocardial infarction) information about the study population. We excluded from the study that the direct cause of death was heart disease and apparent calcification of the mitral valve and/or tricuspid valve. We had no information regarding a past history of heart disease among the cadaver hearts used in this study and, therefore, did not assess coagulation factors related to thrombus formation. Second, absolute values of LAA and RAA measurements were influenced by the fact that all hearts were postmortem specimens, and thus, the unavoidable effect of shrinking as well as the missing intra-atrial pressure, which reduced the diameter and volume of all cardiac structures including atrial appendages, must be taken into consideration. Another limitation is the unavailability of the right and left atrial diameters, which would have provided an insight into the association between the size of the appendages and the size and morphology of the corresponding atria.

\section{Conclusions}

The $3 \mathrm{D}$ structures obtained in this study revealed that RAA is suitable for more favorable blood flow, which may be a reason why thrombus formation is less common in RAA than LAA. In contrast, our results also revealed that the volume, neck area, and amount of PMs of LAA reflected the shape of RAA. Hence, TEE of RAA may be useful in patients at highrisk of thrombus formation in LAA. In addition, these results suggested that measuring LAA was important to predict the possibility of future thrombus formation in RAA. To validate our findings, further studies with a larger number of autopsied hearts with and without AF are warranted.

\section{Abbreviations \\ 2D: Two-dimensional \\ 3D: Three-dimensional \\ AA: Atrial appendages \\ AF: Atrial fibrillation \\ LAA: Left atrial appendage \\ MDCT: Multidetector computed tomography \\ RAA: Right atrial appendage \\ PMs: Pectinate muscles \\ TEE: Transesophageal echocardiography.}

\section{Competing Interests}

The authors declare that they have no conflict of interests.

\section{Authors' Contributions}

Koichi Shinoda, Shogo Hayashi, and Takashi Nakano participated in this study design. Koichi Shinoda, Shogo Hayashi, Ryo Torii, and Takashi Nakano participated in the dissection of the cadavers. Daisuke Fukuoka and Tsuneo Watanabe were in charge of interpreting the data analysis. Daisuke Fukuoka, Tsuneo Watanabe, Shogo Hayashi, and Koichi Shinoda performed statistical analyses. Koichi Shinoda and Shogo Hayashi prepared the first draft of the manuscript. All authors participated in the revision of the manuscript and approved the final paper.

\section{Acknowledgments}

The authors give their heartfelt appreciation to the body donors and their families. We also gratefully thank the Gifu University Hospital Radian Part staff who performed MDCT of the molds.

\section{References}

[1] T. W. Sadler, "Cardiovascular system," in Langman's Medical Embryology, pp. 175-217, LWW, 13th edition, 2015.

[2] C. Chapeau, J. Gutkowska, P. W. Schiller et al., "Localization of immunoreactive synthetic atrial natriuretic factor (ANF) in the heart of various animal species," Journal of Histochemistry and Cytochemistry, vol. 33, no. 6, pp. 541-550, 1985. 
[3] J. M. Stewart, R. Dean, M. Brown et al., "Bilateral atrial appendectomy abolishes increased plasma atrial natriuretic peptide release and blunts sodium and water excretion during volume loading in conscious dogs," Circulation Research, vol. 70, no. 4, pp. 724-732, 1990.

[4] N. M. Al-Saady, O. A. Obel, and A. J. Camm, "Left atrial appendage: structure, function, and role in thromboembolism," Heart, vol. 82, no. 5, pp. 547-554, 1999.

[5] B. D. Hoit, Y. Shao, L. M. Tsai, R. Patel, M. Gabel, and R. A. Walsh, "Altered left atrial compliance after atrial appendectomy. Influence on left atrial and ventricular filling," Circulation Research, vol. 72, no. 1, pp. 167-175, 1993.

[6] M. Bilge, B. Eryonucu, N. Güler, and R. Erkoç, "Right atrial appendage function in patients with chronic nonvalvular atrial fibrillation," Japanese Heart Journal, vol. 41, no. 4, pp. 451-462, 2000.

[7] M. de Divitiis, H. Omran, R. Rabahieh et al., "Right atrial appendage thrombosis in atrial fibrillation: its frequency and its clinical predictors," American Journal of Cardiology, vol. 84, no. 9, pp. 1023-1028, 1999.

[8] A. L. Klein, R. A. Grimm, I. W. Black et al., "Cardioversion guided by transesophageal echocardiography: The ACUTE Pilot Study," Annals of Internal Medicine, vol.126, no. 3, pp. 200209, 1997.

[9] A. L. Klein, R. A. Grimm, R. D. Murray et al., "Use of transesophageal echocardiograpy to guide cardioversion in patients with atrial fibrillation," The New England Journal of Medicine, vol. 344, no. 19, pp. 1411-1420, 2001.

[10] W. J. Manning, D. I. Silverman, S. P. F. Gordon, H. M. Krumholz, and P. S. Douglas, "Cardioversion from atrial fibrillation without prolonged anticoagulation with use of transesophageal echocardiography to exclude the presence of atrial thrombi," The New England Journal of Medicine, vol. 328, no. 11, pp. 750755, 1993.

[11] W. J. Manning, D. I. Silverman, C. S. Keighley, P. Oettgen, and P. S. Douglas, "Transesophageal echocardiographically facilitated early cardioversion from atrial fibrillation using short-term anticoagulation: final results of a prospective 4.5-year study," Journal of the American College of Cardiology, vol. 25, no. 6, pp. 1354-1361, 1995.

[12] V. Rozenberg, F. Boccara, B. Benhalima, N. Lamisse, B. Buyukoglu, and A. Cohen, "Comparison of echocardiographic markers of embolism in atrial flutter and fibrillation: frequency of protruding atherosclerotic plaques in the thoracic aorta," Echocardiography, vol. 17, no. 6, pp. 555-562, 2000.

[13] T. Sahin, D. Ural, T. Kilic et al., "Right atrial appendage function in different etiologies of permanent atrial fibrillation: a transesophageal echocardiography and tissue Doppler imaging study," Echocardiography, vol. 27, no. 4, pp. 384-393, 2010.

[14] B. Subramaniam, M. F. Riley, P. J. Panzica, and W. J. Manning, "Transesophageal echocardiographic assessment of right atrial appendage anatomy and function: comparison with the left atrial appendage and implications for local thrombus formation," Journal of the American Society of Echocardiography, vol. 19, no. 4, pp. 429-433, 2006.

[15] A. Mügge, H. Kühn, P. Nikutta, J. Grote, J. A. Lopez, and W. G. Daniel, "Assessment of left atrial appendage function by biplane transesophageal echocardiography in patients with nonrheumatic atrial fibrillation: identification of a subgroup of patients at increased embolic risk," Journal of the American College of Cardiology, vol. 23, no. 3, pp. 599-607, 1994.
[16] G. Ernst, C. Stöllberger, F. Abzieher et al., "Morphology of the left atrial appendage," Anatomical Record, vol. 242, no. 4, pp. 553-561, 1995.

[17] N. Ikeda, A. Yoshimura, M. Hagiwara, S. Akata, and H. Saji, "Three dimensional computed tomography lung modeling is useful in simulation and navigation of lung cancer surgery," Annals of Thoracic and Cardiovascular Surgery, vol. 19, no. 1, pp. $1-5,2013$.

[18] T. F. Cianciulli, M. C. Saccheri, J. A. Lax et al., "Right and left atrial appendage function in patients with mitral stenosis and sinus rhythm," International Journal of Cardiovascular Imaging, vol. 25, no. 4, pp. 363-370, 2009.

[19] M. Sonka, V. Hlavac, and R. Boyle, Image Processing, Analysis and Machine Vision, Cengage Learning, Boston, Mass, USA, 4th edition, 2014.

[20] K. Yamaji, S. Fujimoto, C. Yutani, T. Hashimoto, and S. Nakamura, "Is the site of thrombus formation in the left atrial appendage associated with the risk of cerebral embolism?" Cardiology, vol. 97, no. 2, pp. 104-110, 2002.

[21] "Cardiogenic brain embolism. Cerebral Embolism Task Force," Archives of Neurology, vol. 43, no. 1, pp. 71-84, 1986.

[22] C. Pollick and D. Taylor, "Assessment of left atrial appendage function by transesophageal echocardiography. Implications for the development of thrombus," Circulation, vol. 84, no. 1, pp. 223-231, 1991.

[23] H. Kato, M. Nakanishi, N. Maekawa, T. Ohnishi, and M. Yamamoto, "Evaluation of left atrial appendage stasis in patients with atrial fibrillation using transesophageal echocardiography with an intravenous albumin-contrast agent," American Journal of Cardiology, vol. 78, no. 3, pp. 365-369, 1996.

[24] D. N. Rubin, S. E. Katz, M. F. Riley, P. S. Douglas, and W. J. Manning, "Evaluation of left atrial appendage anatomy and function in recent-onset atrial fibrillation by transesophageal echocardiography," American Journal of Cardiology, vol. 78, no. 7, pp. 774-778, 1996.

[25] J. P. Veinot, P. J. Harrity, F. Gentile et al., "Anatomy of the normal left atrial appendage: a quantitative study of age-related changes in 500 autopsy hearts: implications for echocardiographic examination," Circulation, vol. 96, no. 9, pp. 3112-3115, 1997.

[26] L. Di Biase, P. Santangeli, M. Anselmino et al., "Does the left atrial appendage morphology correlate with the risk of stroke in patients with atrial brillation? Results from a multicenter study," Journal of the American College of Cardiology, vol. 60, no. 6, pp. 531-538, 2012.

[27] T. Kimura, S. Takatsuki, K. Inagawa et al., "Anatomical characteristics of the left atrial appendage in cardiogenic stroke with low CHADS2 scores," Heart Rhythm, vol. 10, no. 6, pp. 921-925, 2013.

[28] O. Ozer, I. Sari, and V. Davutoglu, "Right atrial appendage: forgotten part of the heart in atrial fibrillation," Clinical and Applied Thrombosis/Hemostasis, vol. 16, no. 2, pp. 218-220, 2010. 

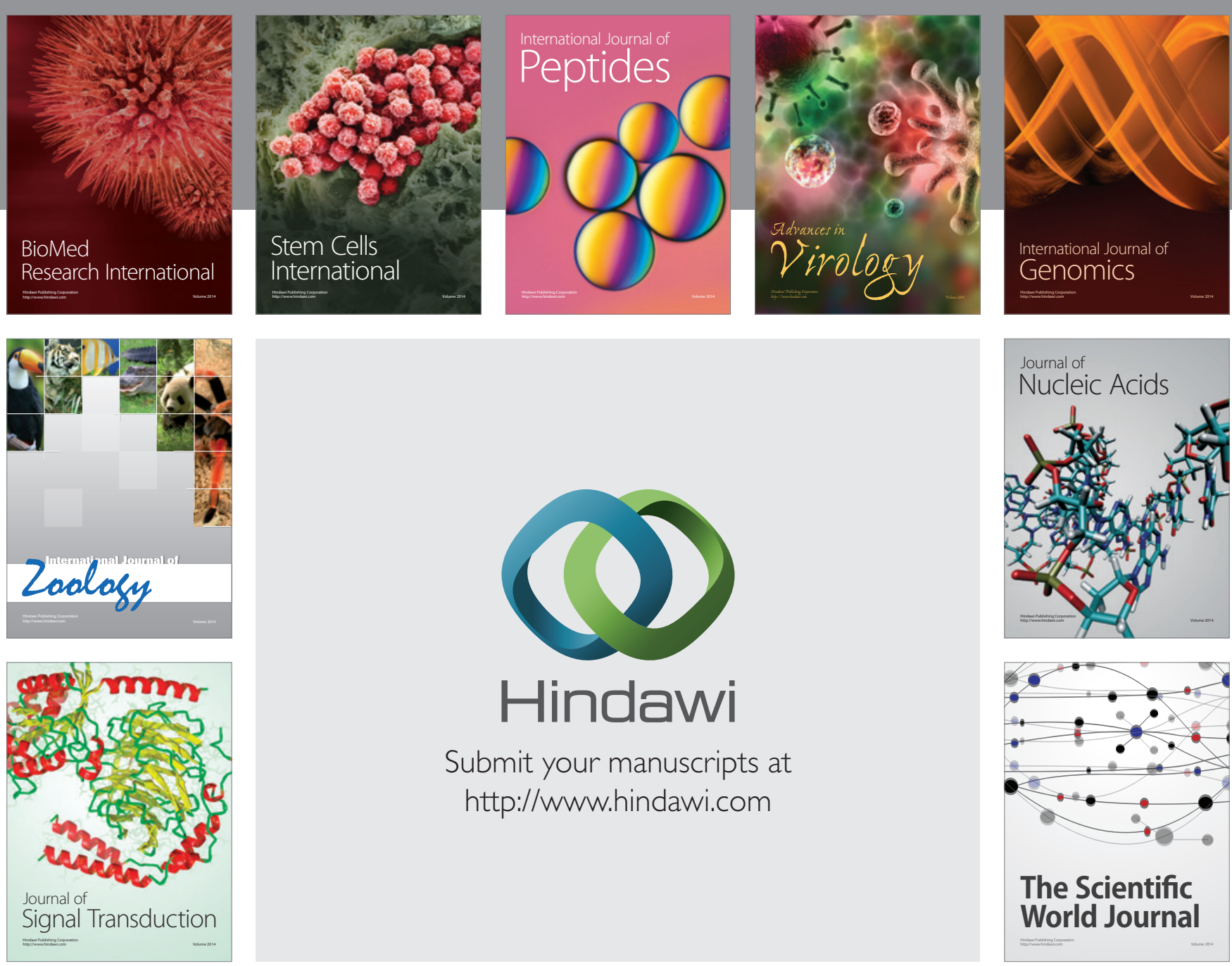

Submit your manuscripts at

http://www.hindawi.com
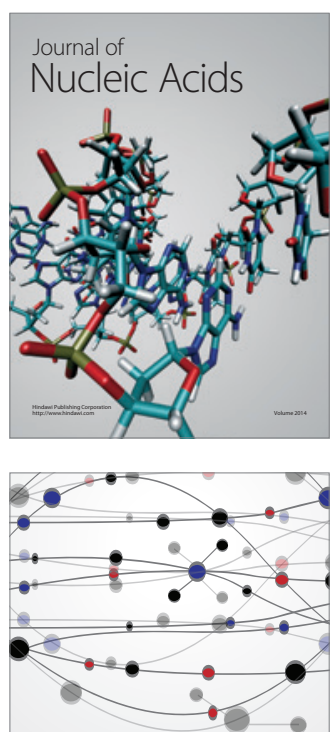

The Scientific World Journal
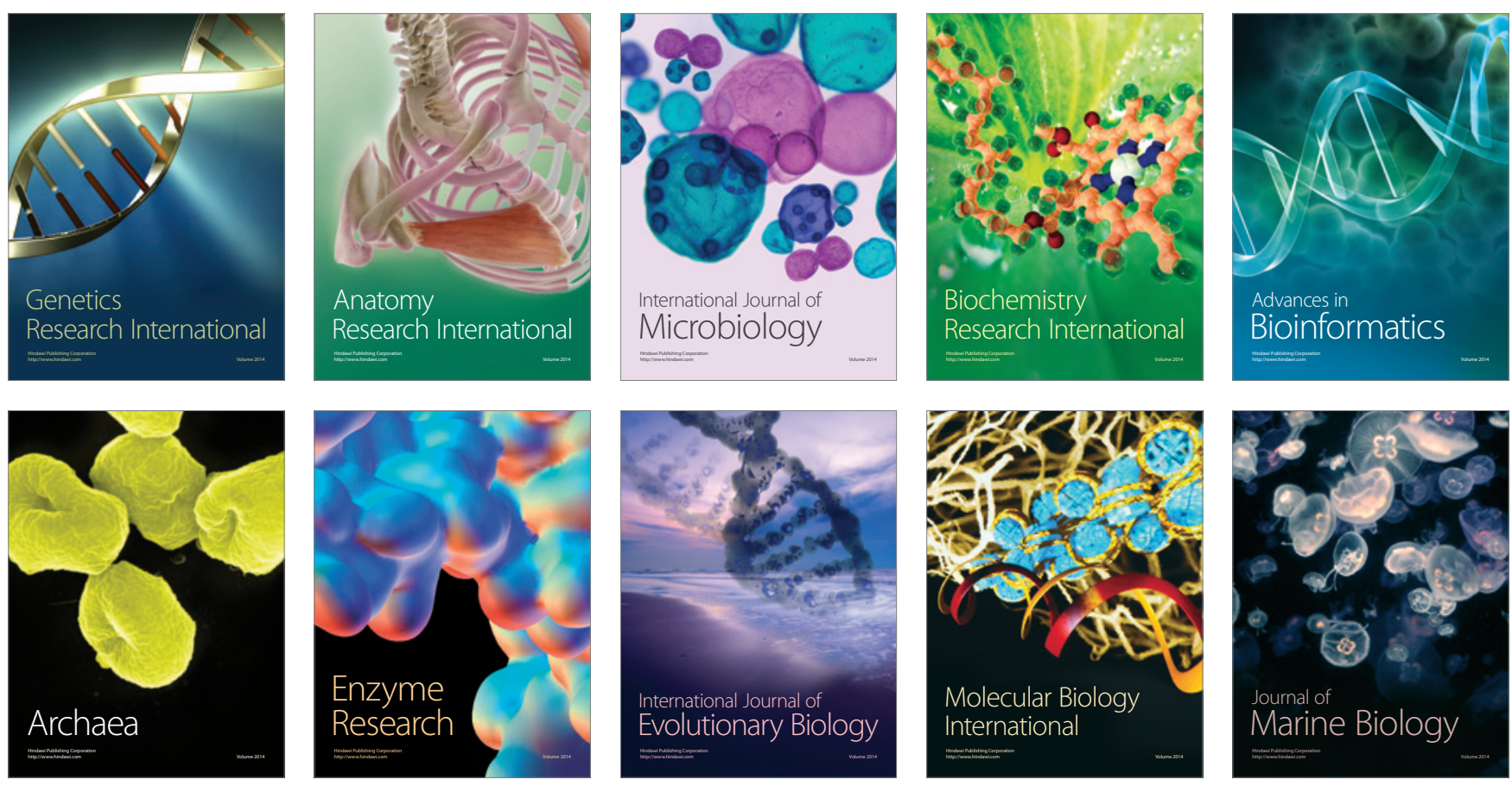